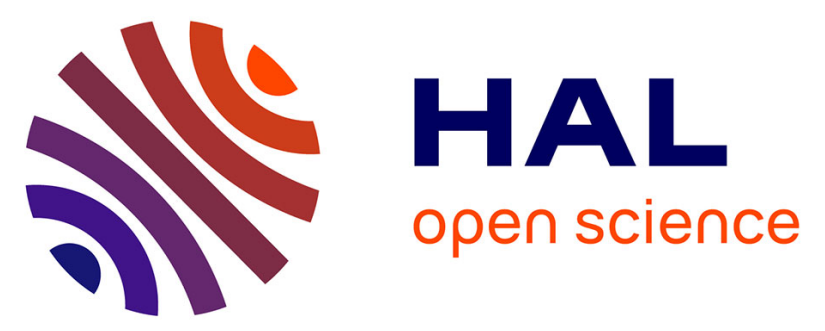

\title{
Etude comparative par indentation des caractéristiques mécaniques de verres sodo-calcique et vitrocéramique rodés et polis par des grains abrasifs liés
}

\author{
Mohamed Bentoumi, Djamel Bouzid, Isabel Hervas, Alain Iost
}

\section{To cite this version:}

Mohamed Bentoumi, Djamel Bouzid, Isabel Hervas, Alain Iost. Etude comparative par indentation des caractéristiques mécaniques de verres sodo-calcique et vitrocéramique rodés et polis par des grains abrasifs liés. Matériaux \& Techniques, 2015, 103 (6), pp.14. 10.1051/mattech/2015060 . hal01313307

\author{
HAL Id: hal-01313307 \\ https://hal.science/hal-01313307
}

Submitted on 9 May 2016

HAL is a multi-disciplinary open access archive for the deposit and dissemination of scientific research documents, whether they are published or not. The documents may come from teaching and research institutions in France or abroad, or from public or private research centers.
L'archive ouverte pluridisciplinaire HAL, est destinée au dépôt et à la diffusion de documents scientifiques de niveau recherche, publiés ou non, émanant des établissements d'enseignement et de recherche français ou étrangers, des laboratoires publics ou privés. 


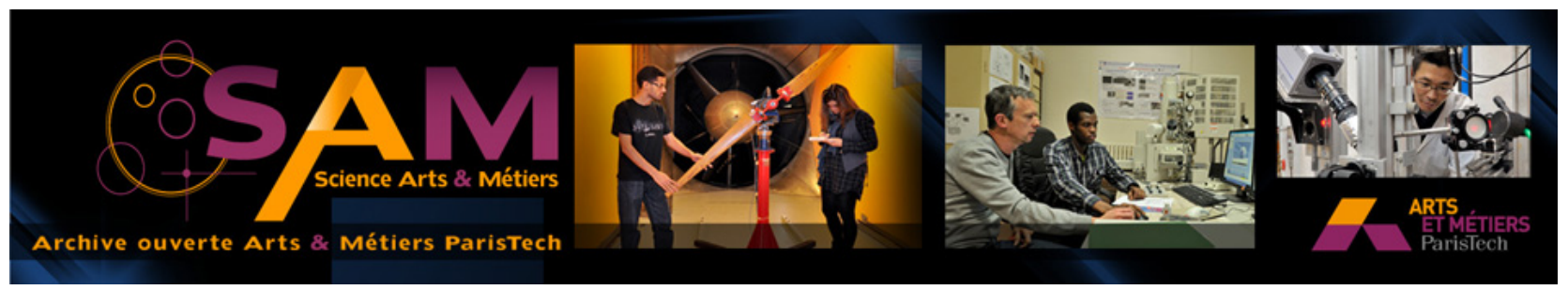

\section{Science Arts \& Métiers (SAM)}

is an open access repository that collects the work of Arts et Métiers ParisTech researchers and makes it freely available over the web where possible.

This is an author-deposited version published in: http://sam.ensam.eu

Handle ID: .http://hdl.handle.net/10985/10774

\section{To cite this version :}

Mohamed BENTOUMI, Djamel BOUZID, Isabel HERVAS, Alain IOST - Etude comparative par indentation des caractéristiques mécaniques de verres sodo-calcique et vitrocéramique rod'es et polis par des grains abrasifs liés - Matériaux \& Techniques - Vol. 103, n6, p.14 - 2015 


\title{
Étude comparative par indentation des caractéristiques mécaniques de verres sodo-calcique et vitrocéramique rodés et polis par des grains abrasifs liés
}

\author{
Mohamed Bentoumi $^{1}$, Djamel Bouzid ${ }^{1}$, Isabel Hervas ${ }^{2}$ et Alain Iost $^{2}$
}

\begin{abstract}
Résumé - Malgré le nombre important de paramètres technologiques mis en jeux, le polissage des matériaux fragiles par des grains abrasifs liés montre une grande efficacité et une qualité de surface meilleure par rapport au polissage par des grains abrasifs libres. Cette méthode permet d'obtenir des rugosités $R a$ et $R q$ de l'ordre de 30 à $40 \mathrm{~nm}$ sur les verres étudiés. Les essais d'indentation instrumentée ont été effectués en faisant varier les charges de 5 à $40 \mathrm{~N}$ avec une bonne reproductibilité. Pour le verre céramique on observe des pop-in à partir de $50 \mathrm{~N}$, alors que ces derniers n'apparaissent qu'à partir de $100 \mathrm{~N}$ sur les verres flint lourd et flotté, et qu'ils ne sont pas mis en évidence sur le verre crown. La ténacité a été calculée à partir de la longueur de fissures formées par différentes relations en fonction des hypothèses faites sur la géométrie des fissures. Les résultats obtenus sur ces différents verres, ainsi que leurs dispersions, sont comparés.
\end{abstract}

Mots clés : Verre / polissage / indentation / ténacité

Abstract - Comparative study by indentation test of the mechanical characteristics of sodalime and ceramic glasses ground and polished by bound abrasive grains. In this work, indentation toughness of 4 optical glasses (Crown K5, Heavy flint, Float glass and Zerodur ${ }^{\circledR}$ ) was analyzed regarding its surface quality. Despite the large number of the involved technological parameters, polishing of brittle materials by abrasive bonded grains shows a high efficiency and a better surface quality compared to polishing by free abrasive grains. This method has allowed obtaining roughness parameters, $R a$ and $R q$, about 30-40 nm. Instrumented indentation tests were performed by varying the load from 5 to 300 N. A good reproducibility was achieved. Pop-in in glass ceramic was observed from 50 N, whereas it only appears from $100 \mathrm{~N}$ on heavy flint and float glasses and they are not highlighted on crown glass. Indentation toughness was calculated by means of the cracks length using different relationships based on hypotheses concerning the cracks geometry. Anstis, Tanaka, Niihara and Laugier equations were employed. Finally, the results obtained from these equations and their dispersions are compared.

Key words: Glass / polishing / indentation / toughness

\section{Introduction}

La caractérisation des propriétés mécaniques par indentation fait l'objet d'un intérêt scientifique important pour les matériaux fragiles tels que les céramiques et les verres, (céramiques ou métalliques) et les composés intermétalliques. La technique d'indentation est souvent un instrument de base largement répandu dans les laboratoires industriels et de recherches. Les différentes méthodes utilisées pour estimer la ténacité par indentation ont fait l'objet de nombreux travaux publiés. Les équations pour les matériaux fragiles utilisent la mesure des fissures formées par l'indenteur ou les décrochements, pop-in, observés sur la courbe charge-déplacement [1] quand l'essai est instrumenté. Les différentes équations sont établies en comparant ces résultats à la ténacité obtenue par les essais d'indentation classique.

Les méthodes utilisées en indentation permettent généralement de déterminer la ténacité $K_{I c}$ par

\footnotetext{
1 Institut Optique et Mécanique de Précision, LOA, Ferhat Abbas, Sétif, Algérie

mohamedbentoumi709@gmail.com

2 Arts et Métiers ParisTech, MSMP, Lille, France
} 

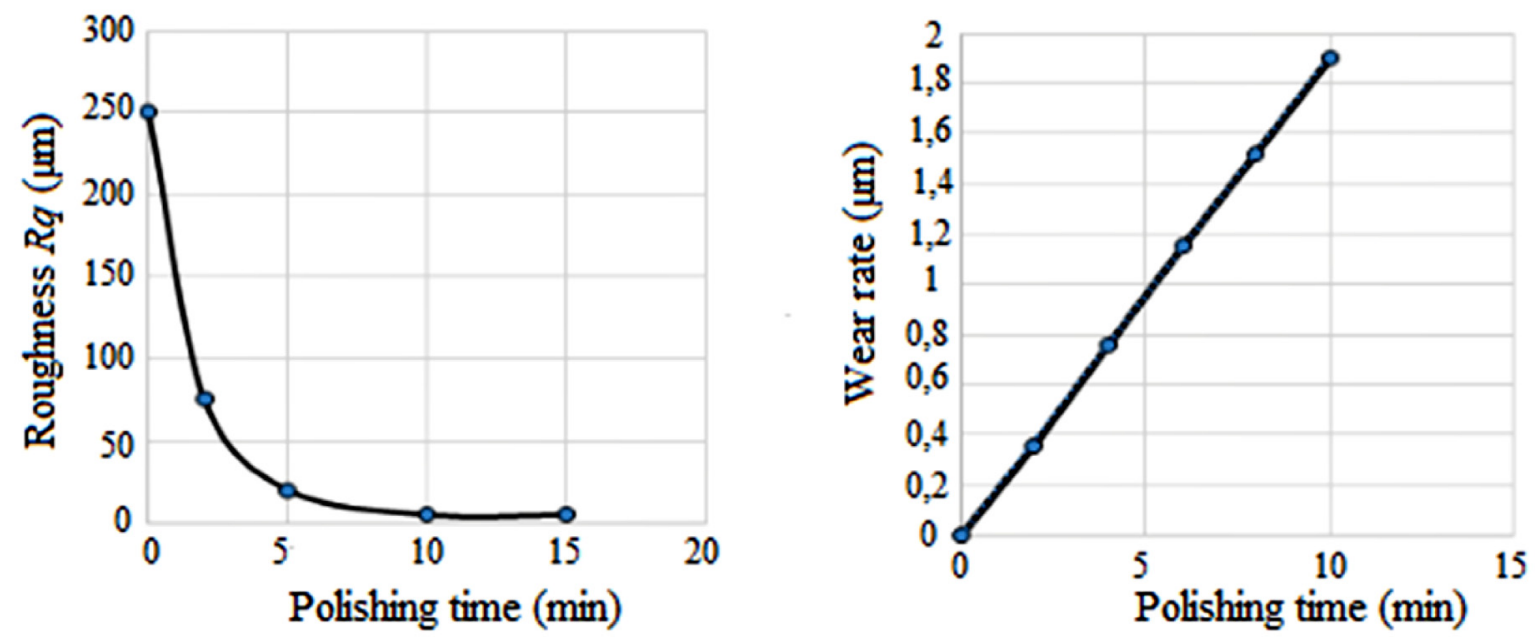

Fig. 1. Évolution de la rugosité et du taux d'enlèvement de matière en fonction du temps de polissage.

Fig. 1. Evolution of roughness and removal material rate of as a function of polishing time.

indentation avec une incertitude pouvant aller jusqu'à $50 \%$ [2]. La caractérisation des matériaux dépend de l'état de surface donc de la méthode de préparation (rodage et polissage) et des défauts produits par cette dernière. Les procédés de finition classique restent de nos jours les plus fiables pour la réalisation de surface de très haute qualité, mais ils dépendent d'un nombre important de paramètres technologiques (les agents de rodage et de polissage) qui ont une influence sur l'état de surface (rugosité et écrouissage) et entrainent une dispersion des résultats.

Le rodage par des grains abrasifs libres utilise des poudres mélangées avec de l'eau pour former l'agent de polissoir. L'opération est réalisée sur un rodoir en acier ou en bronze [3-5]. Durant le contact, ce processus conduit à des fissurations locales de la couche superficielle du matériau avec un petit enlèvement de copeaux [6]. Le rodage par des grains abrasifs liés permet d'utiliser des outils métalliques, plans ou sphériques, dont la surface utile contient des grains abrasifs tandis que l'eau utilisée sert à refroidir l'outil, dégager les débris et aider la circulation des particules entre l'échantillon et l'outil.

En étudiant l'état de surface d'un dioptre en verre, Rupp [7] a montré que ce processus est compliqué comparé à celui qui utilise un abrasif libre. Le rodage par abrasifs liés est un processus pendant lequel l'orientation des grains abrasifs vers la surface de l'échantillon est constante. Si l'outil est neuf, les grains pénètrent profondément dans le verre et produisent de petites écailles et des fêlures. Des composants optiques de haute précision ont été obtenus par ces méthodes. À titre d'exemple, des surfaces sphériques convexes et concaves ont été fabriquées à l'université de Rochester (New York USA) avec un rayon de courbure de $5 \mathrm{~mm}$ à l'infini et des diamètres de travail de 10 à $150 \mathrm{~mm}$; après moins de 5 minutes de micro-rodage, la surface spéculée résultante avait une rugosité quadratique $(R q)$ inférieure à $20 \mathrm{~nm}$ [8-13]. Les taux d'enlèvement de matière typiques sont de 6 à $10 \mu \mathrm{m} / \mathrm{min}$ pour une taille des abrasifs en diamant de 2 à $4 \mu \mathrm{m}$. Bouzid et al. [14] ont employé des outils abrasifs liés à base d'oxyde de cérium pour le polissage des verres optique BK7. Ils ont étudié l'influence du temps de polissage sur la rugosité de la surface et le taux d'enlèvement de matière (Fig. 1). Ces auteurs ont remarqué que la rugosité diminue rapidement pendant les 5 premières minutes, qu'elle atteint ensuite un minimum pour $10 \mathrm{~min}$ avant d'augmenter à nouveau. Par contre le taux d'enlèvement de matière varie linéairement en fonction du temps de polissage.

Concernant le polissage illustré sur la Figure 2, le principe est le même que pour le rodage sauf que l'agent polissoir est plus doux et les grains abrasifs plus fins. Marioge [15] conclu que le taux d'enlèvement de matière et la rugosité sont proportionnels à la grosseur des grains. En effet, le polissage avec des grains abrasifs plus fins réduit la rugosité de la surface avec un faible taux d'enlèvement de matière. Cependant, la transition entre le rodage et le polissage est étroit, puisque le rodage peut aussi être utilisé sous certaines conditions comme opération de semi-finition. Une rugosité inférieure à $1 \mathrm{~nm}$ et un écart de forme de l'ordre du nanomètre peuvent être atteints en polissage des surfaces planes et sphérique. Le verre céramique du type Zerodur ${ }^{\circledR}$ Schott peut même atteindre une rugosité $(R q)$ de l'ordre $02 \mathrm{~nm}$ [16]. Le polissage se caractérise par la génération d'une rugosité minimale de la surface avec un faible taux d'enlèvement de matière. Des grains abrasifs de taille micrométriques et des polissoirs relativement doux sont généralement utilisés.

La détermination de la ténacité par indentation nécessite un matériau avec une surface plane, polie et un équipement expérimental adéquat pour mesurer la taille des fissures formées.

De nombreuses relations ont été développées pour exprimer la ténacité en fonction du type de fissure. Ponton et Rawlings [19,20] ont recensé les différents modèles existant à l'époque en fonction du type de fissures : Palmqvist (P), half-penny ou Médiane (M) (Fig. 3). 


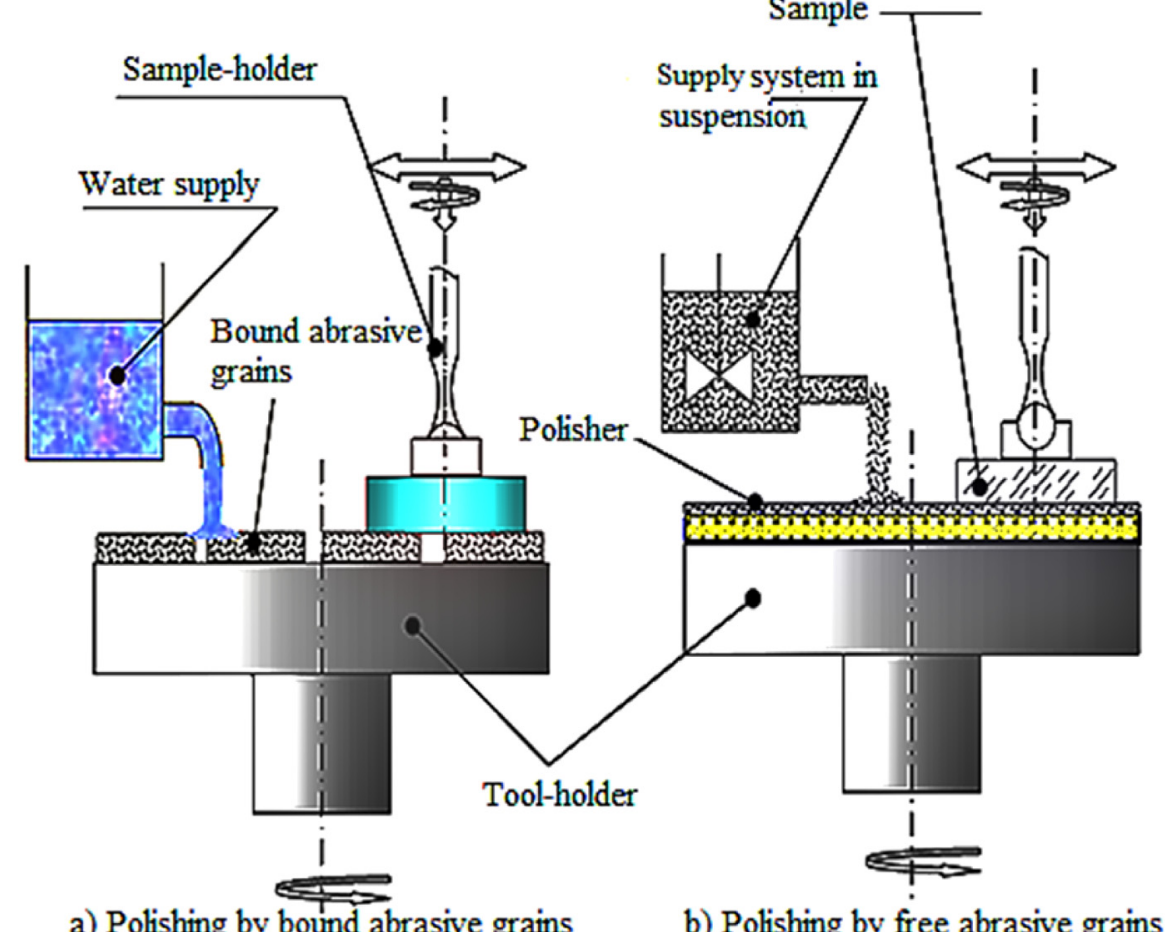

a) Polishing by bound abrasive grains

b) Polishing by free abrasive grains

Fig. 2. Principe du rodage et du polissage du verre par des grains abrasifs libres ou liés $[17,18]$.

Fig. 2. Principle of glass ground and polishing by free or bound abrasive grains [17, 18].

Souvent les fissures sont de type $\mathrm{P}$ pour les faibles charges appliquées (charges relatives en fonction de la ténacité du matériau) et deviennent du type $\mathrm{M}$ quand les charges augmentent.

La limite entre les deux types de fissure correspond à une valeur critique du rapport $c / a$. En règle générale, si $c / a \geq 2$, le modèle de fissuration est considéré être de type Médian (half-penny), et si $c / a<2$, le modèle de fissuration est considéré comme étant de Palmqvist. Cependant pour d'autres auteurs la limite est $c / a=3$ ou $2,5[21,22]$.

Dans cet article nous présentons les résultats comparatifs obtenus pour les verres fragiles : Crown K5, Flint lourd, verre Flotté et Zerodur ${ }^{\circledR}$ dont la surface est obtenue après rodage par des grains abrasifs en oxyde d'aluminium $\mathrm{Al}_{2} \mathrm{O}_{3}$, puis polissage par des grains abrasifs en oxyde de cérium $\mathrm{CeO}_{2}$ libres ou liés. L'état de surface (rugosité) des matériaux fragiles est obtenu au moyen d'un profilomètre optique. La ténacité $K_{I c}$ est déterminée à l'aide des différentes relations établies.

\section{Méthodes expérimentales}

\subsection{Matériaux}

Les matériaux utilisés sont des verres minéraux fragiles à base de silice. Les verres Sodocalciques sont des verres les plus répandus, ils sont utilisés dans l'industrie pour la fabrication industrielles 'a usage alimentaire : bouteilles, flacons et des verres 'a boire mais aussi des verres plats (flotté) pour le vitrage industriel : double vitrage (bâtiments) et vitrage feuilleté ou trempé (pare-brise de véhicule) de protection solaire. La technologie du verre «float» a été découverte par Pilkington Brothers dans les années 1950 [23]. Le verre flotté teinté dans la masse grâce 'a des colorants (oxydes métalliques) ajoutés 'a la composition du float. Il a une qualité de surface excellente, transparente et lisse, résiste aux chocs thermiques et possède un taux d'absorption énergétique très élevé.

Les crowns K5 sont les verres les plus utilisés en conception optique Une lentille convexe en crown et une lentille concave en flint réalisent les premiers doublets optiques pour corriger l'aberration chromatique ou sphérique; leurs compositions restent proches de celle du verre flotté avec une forte teneur en silice, en soude et en calcium. Les verres crown ont un faible indice de réfraction et un nombre d'Abbe élevé supérieure à 50 .

Les flints KzSFN5 très riches en plomb, ont un fort indice de réfraction et sont très dispersifs par rapport aux verres précédents Ils sont utilisés généralement dans la verrerie d'art pour leur brillance, dans le domaine des verres optiques et dans la protection des écrans.

Les verres Silico-boro-calciques ou vitrocéramiques de type Zerodur ${ }^{\circledR}$ sont constitués de microcristaux dispersés 


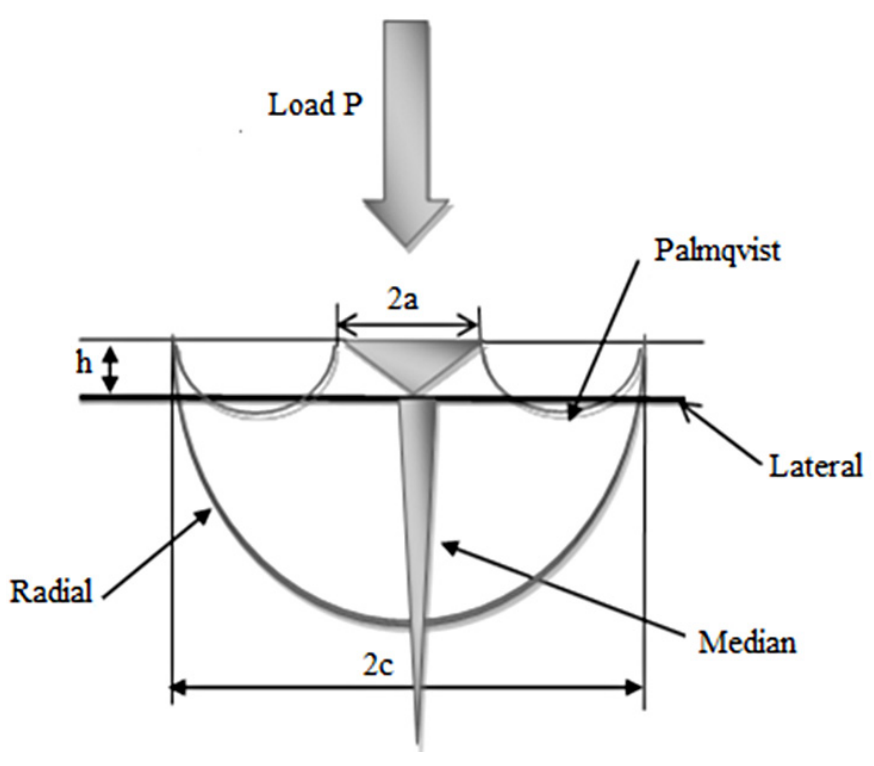

Fig. 3. Représention schématique des systèmes de fissures induits par indentation Vickers.

Fig. 3. Schematic representation of the crack systems for a Vickers indentation.

dans une phase vitreuse Le Zerodur ${ }^{\circledR}$ de Schott reste relativement transparent à l'œil car les indices de réfractions de la matrice vitreuse et de la phase cristallisée sont proches; cependant la dispersion créée par la présence de nano cristaux empêche son utilisation dans des optiques passives, c'est-à-dire pour transmettre la lumière [24-26]. Les vitrocéramiques résistent à des chocs thermiques exceptionnellement élevés, supérieurs à $300 \mathrm{~K}$, et possèdent un faible coefficient de dilatation. Ses utilisations les plus connues sont les plaques de cuisson et la réalisation de grands miroirs astronomiques.

Les verres utilisés sont de dimension rectangulaire $32 \times$ $23 \times 5 \mathrm{~mm}^{2}$ pour le crown et $32 \times 23 \times 6 \mathrm{~mm}^{2}$ pour le flint de dimension carrée $44 \times 44 \times 8 \mathrm{~mm}^{2}$ pour le verre flotté et sous forme de cylindre $25 \times 12 \mathrm{~mm}^{2}$ pour le Zerodur ${ }^{\circledR}$.

Les compositions chimiques et physiques des quatre nuances déterminées par diffraction des rayons X et par mesure de l'indice de réfraction (Réfractomètre Abbe de Carl Zeiss) sont présentées dans le Tableau 1.

\section{2 Élaboration des grains}

L'abrasif utilisé en rodage est à base d'alumine sous forme de grains liés par un liant organique ou vitrifiant. La préparation consiste à mélanger $80 \%$ d'alumine et $20 \%$ de liant (résine, verre, polyuréthane) pendant 5 à $10 \mathrm{~min}$ pour rendre le mélange homogène. Les teneurs en grains abrasifs et en liant dans le mélange ont été déterminées par des essais préliminaires. La mise en forme réalisée au niveau de l'institut optique et mécanique de précision de l'université de Sétif, consiste à soumettre une quantité de mélange $(0,720 \mathrm{~g})$ déposée dans une matrice fermée à une compression sous une pression variant entre 100 et $300 \mathrm{MPa}$ ce qui correspond à des efforts variant de 5 à $15 \mathrm{KN}$ par pas de $5 \mathrm{KN}$. Les grains abrasifs liés sont ensuite chauffés à une température inférieure à celle de fusion, pendant une durée de 30 à $60 \mathrm{~min}$. La température de cuisson est choisie selon le type de liant utilisé : $150^{\circ} \mathrm{C}$ pour la résine, $850{ }^{\circ} \mathrm{C}$ pour le verre et $120^{\circ} \mathrm{C}$ pour le polyuréthane.

\subsection{Rodage et polissage}

L'opération de rodage a été effectuée sur une machine classique, constituée d'un porte outil qui tourne à une vitesse de rotation de $124 \mathrm{tr} / \mathrm{min}$ Les grains abrasifs liés sont collés sur le porte outil qui tourne en sens inverse de l'échantillon de verre fixé sur le porte pièce par des résines. Ce dernier tourne à une vitesse constante et oscille à des amplitudes réglables selon le diamètre du rodoir. Les grains abrasifs utilisés sont du type $\alpha$ alumine $\left(\mathrm{Al}_{2} \mathrm{O}_{3}\right)$ (Abralox, qualité E, de la société Pieplow et Brandt Gmbh). Les trois types de verre : Céramique (Zerodur ${ }^{\circledR}$ ) Crown K5 et Flint lourd de Schott sont polis successivement par différentes tailles de grains : 80 40, 15 12 et $9 \mu \mathrm{m}$, pendant une durée de $60 \mathrm{~min}$, puis à l'aide d'un polissoir plus doux et des grains abrasifs d'oxyde de cérium (CEROX 1660-1663). Le polissage permet d'obtenir une surface transparente, la diminution de la rugosité et la mise en forme à la précision nécessaire (Fig. 4). Le temps de polissage des verres crown et flints varie de 0 à 15 min par contre le Zerodur ${ }^{\circledR}$ difficile à polir, nécessite un temps de polissage important pouvant aller jusqu'à $45 \mathrm{~min}$.

\section{4 État de surface}

La rugosité est étudiée par le profilomètre optique Veeco Wyko NT9300 La rugosité obtenue pour le verre flint lourd est satisfaisante comparée à celle du crown

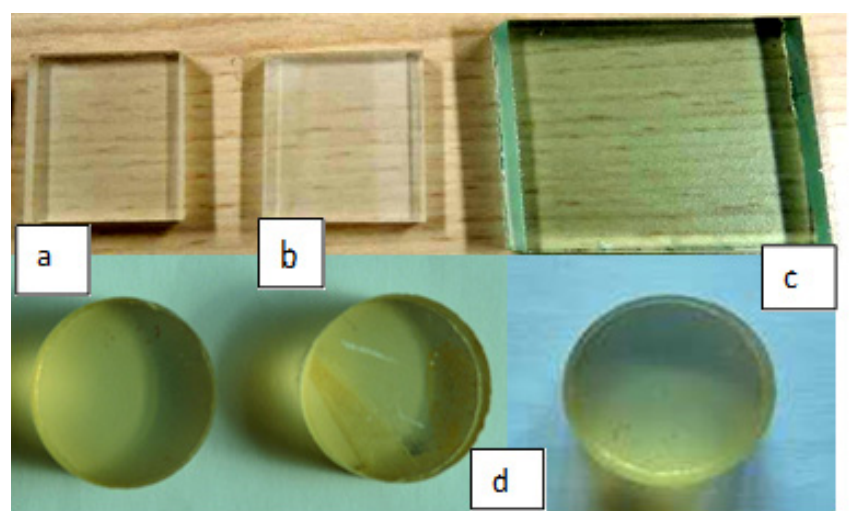

Fig. 4. Représentation des échantillons rodés et polis par des grains abrasifs liés flint lourd (a), crown K5 (b), verre flotté non rodé ni poli (c) et Zerodur ${ }^{\circledR}(\mathrm{d})$.

Fig. 4. Ground and polished glasses with bound abrasive grains: (a) heavy flint, (b) crown K5, (c) float glass (not ground or polished) and (d) Zerodur ${ }^{\circledR}$. 
Tableau 1. Composition chimique des matériaux utilisés $\left(\rho:\right.$ Densité $\left(\mathrm{gr} / \mathrm{cm}^{3}\right), n:$ Indice de réfraction, $V:$ Nombre d'Abbe et $\nu$ : Coefficient de poisson).

Table 1. Chemical composition of the glasses ( $\rho$ : density $\left(\mathrm{gr} / \mathrm{cm}^{3}\right), n$ : Refractive index, $V$ : Abbe number and $\nu$ : Poisson's coefficient).

\begin{tabular}{|c|c|c|c|c|c|c|c|c|c|c|c|c|c|c|c|c|}
\hline \multirow[t]{2}{*}{ Glasses } & \multicolumn{4}{|c|}{$\begin{array}{c}\text { Physical } \\
\text { properties }\end{array}$} & \multicolumn{12}{|c|}{ Composition \% } \\
\hline & $\begin{array}{c}\text { Densité } \\
\left(\mathrm{gr} / \mathrm{cm}^{3}\right)\end{array}$ & $\begin{array}{l}\text { Indice de } \\
\text { réfraction }\end{array}$ & $\begin{array}{l}\text { Nombre } \\
\text { d'Abbe }\end{array}$ & $\begin{array}{l}\text { Coefficient } \\
\text { de poisson }\end{array}$ & $\mathrm{SiO}_{2}$ & $\mathrm{Na}_{2} \mathrm{O}$ & $\mathrm{CaO}$ & $\mathrm{K}_{2} \mathrm{O}$ & $\mathrm{MgO}$ & $\mathrm{Al}_{2} \mathrm{O}_{3}$ & $\mathrm{P}_{2} \mathrm{O}_{5}$ & $\mathrm{Li}_{2} \mathrm{O}$ & $\mathrm{TiO}_{2}$ & $\mathrm{ZrO}_{2}$ & $\mathrm{~A}_{s} \mathrm{O}_{3}$ & $\mathrm{Fe}_{2} \mathrm{O}_{3}$ \\
\hline Float & 2,47 & 1,44 & 61,3 & 0,20 & 70,3 & 13,8 & 10 & 0,6 & 4,1 & 1,1 & - & - & - & - & - & 0,1 \\
\hline Crown K5 & 2,5 & 1,52 & 58,6 & 0,22 & 71,7 & 13,5 & 10,2 & 0,73 & 2,77 & 1,1 & - & - & - & - & - & - \\
\hline Heavy flint & 3,67 & 1,65 & 39,6 & 0,28 & 38,9 & - & - & 5,24 & - & - & 55,86 & - & - & - & - & - \\
\hline Zerodur & 2,53 & 1,52 & 56,2 & 0,24 & 55,8 & 0,5 & - & - & 1 & 26,3 & 7,9 & 3,7 & 2,3 & 1,9 & 0,5 & 0,1 \\
\hline
\end{tabular}
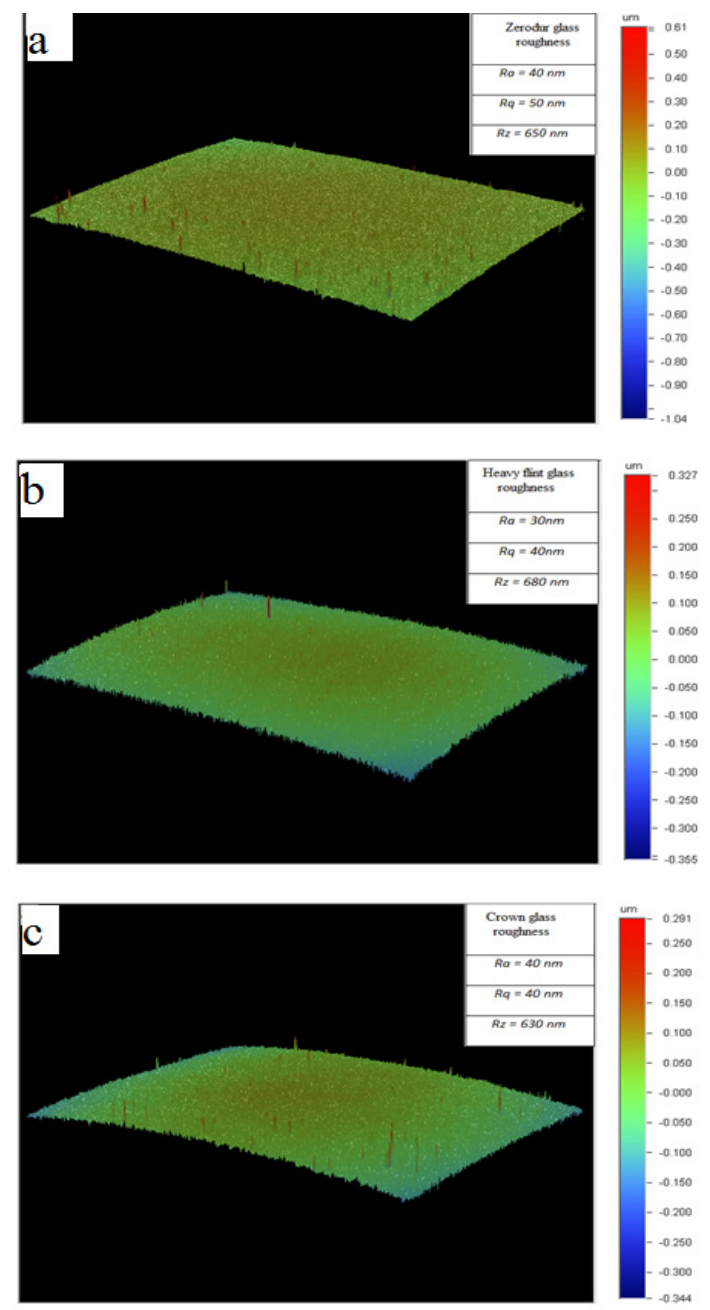

Fig. 5. Rugosité en 3D obtenue pour les trois verres polis par des grains abrasifs liés : Zerodur ${ }^{\circledR}$ (a), Flint (b) et Crown (c).

Fig. 5. 3D Pictures of roughness obtained for the three glasses polished by bound abrasive grains: Zerodur ${ }^{\circledR}$ (a), Heavy flint (b) and Crown K5 (c).

et du Zerodur ${ }^{\circledR}$. L'état de surface est meilleur pour le polissage des verres par des grains abrasifs liés que pour le polissage par les grains abrasifs libres.

Les valeurs de rugosité $R a$ présentées dans la Figure 5 semblent presque identiques pour les trois verres traités :
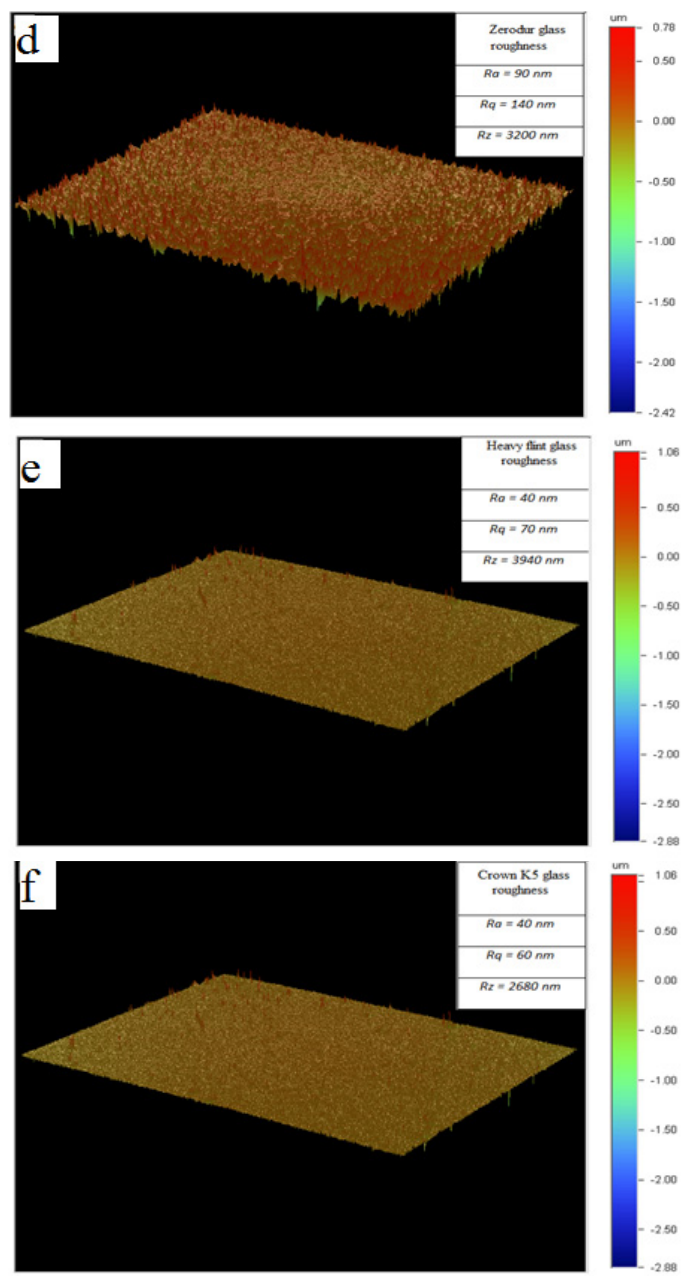

Fig. 6. Rugosité en 3D obtenue pour les trois verres polis par des grains abrasifs libres : Zerodur ${ }^{\circledR}$ (d), Flint (e) et Crown (f). Fig. 6. 3D Pictures of roughness obtained for the three polished glasses by free abrasive grains: Zerodur ${ }^{\circledR}(d)$, heavy Flint (e) and Crown $K 5(f)$.

$R a=30,40$ et $40 \mathrm{~nm} ; R q=40,40$ et $50 \mathrm{~nm}$ respectivement pour flint, crown et Zerodur ${ }^{\circledR}$. Concernant le polissage par grains abrasifs libres, les valeurs de $R a$ mentionnées dans la Figure 6 sont identiques pour le verre crown et flint $(R a=40 \mathrm{~nm})$, et sont de $90 \mathrm{~nm}$ pour 
le Zerodur ${ }^{\circledR}$. Les valeurs de $R q$ semblent presque identiques pour le flint et le crown (70 et $60 \mathrm{~nm}$, respectivement), mais atteignent $140 \mathrm{~nm}$ pour le Zerodur ${ }^{\circledR}$. Ceci peut être expliqué au départ par les différentes dimensions de grains utilisés et les conditions de travail qui ne sont pas identiques par rapport aux autres verres ainsi qu'à la difficulté du polissage des verres céramiques par grains abrasifs libres.

\subsection{Essai d'indentation}

Les essais d'indentation sont effectués à l'aide du macroduromètre instrumenté Zwick ZHU 2.5.

Les charges appliquées permettant de mesurer la ténacité sont de 5 à $40 \mathrm{~N}$.

Des essais d'indentation sous des charges appliquées pouvant atteindre $300 \mathrm{~N}$ ont été menés afin de mettre en évidence des pop-in. La Figure 7 présente l'évolution de la charge en fonction de la pénétration pour les quatre échantillons avec des charges allant de 5 à $90 \mathrm{~N}$.

La ténacité $K_{I C}$ est calculée en utilisant les quatre relations du Tableau 2 : deux pour les fissures de type $\mathrm{M}$ et deux pour les fissures de type $\mathrm{P}$.

Les valeurs moyennes obtenues de $K_{I} c, E$ et $H V$ sont regroupés dans le Tableau 3.

La Figure 8 présente l'évolution des indentations Vickers obtenues sous différentes charges pour les quatre matériaux. On observe clairement l'empreinte suivie des fissurations radiales dès la première charge sélectionnée $(5 \mathrm{~N})$ pour chaque type de verre. Seul le Zerodur ${ }^{\circledR}$ possède une fissure radiale avec une légère déformation de l'empreinte. À partir des charges comprises entre 10 et $15 \mathrm{~N}$, et lors du retrait de pénétrateur, on observe une légère déformation de l'empreinte due au retour élastique pour les quatre matériaux. Cette déformation prend la forme d'une déflection des faces de l'empreinte résiduelle et l'apparition d'un certain nombre d'écailliages. Pour des charges supérieures à $15 \mathrm{~N}$, on constate l'apparition systématique d'écailles. L'empreinte et les fissures sont assez nettes pour le verre plat. Par contre, pour le Zerodur ${ }^{\circledR}$, elles ont tendance à disparaitrent, la taille des défauts augmente, les fissures latérales et radiales s'élargissent et forment un contour fermé plus ou moins régulier. Finalement et pour des charges $40 \mathrm{~N}$, les fissures radiales de l'empreinte s'élargissent pour les deux verres flotté et flint. Pour le crown et le Zerodur ${ }^{\circledR}$ la déformation totale de l'empreinte et l'écaillage se développent progressivement, et leur forme tend à devenir circulaire.

Des charges supérieures à $90 \mathrm{~N}$ ont été appliquées sur ces quatre échantillons afin de visualiser la forme, la nature des fissures et le comportement des matériaux. La Figure 10 présente des empreintes d'indentations pour les quatre verres utilisés. On remarque, durant l'application des charges importantes $(100,200$ et 300 N), le changement du mode de fissuration.
Tableau 2. Equations utilisées pour le calcul de la ténacité $\left(\mathrm{K}_{1 c}\right)$ pour différents hypothèses de géométrie de fissure.

Table 2. Equations used to calculate the fracture toughness $\left(K_{1 c}\right)$ for different crack geometries.

\begin{tabular}{ccc}
\hline No. & Equations & References \\
\hline 1 & $\mathrm{~K}_{I C}=0.016(E / H)^{1 / 2} P C^{-3 / 2}$ & Anstis [26] \\
2 & $\mathrm{~K}_{I C}=0.022(E / H)^{2 / 5} P C^{-3 / 2}$ & Tanaka [27] \\
3 & $\mathrm{~K}_{I C}=0.089(E / H)^{2 / 5}(P / a) C^{-1 / 2}$ & Niihara [28] \\
4 & $\mathrm{~K}_{I C}=0.089(E / H)^{2 / 3}(a / l) P C^{-3 / 2}$ & Laugier [29] \\
\hline
\end{tabular}

\section{Resultats et discussions}

La Figure 7 présente les courbes d'indentation pour les quatre nuances obtenues avec des charges progressives de 5, 10, 15, 20, 30 et $40 \mathrm{~N}$. Les essais montrent une assez bonne reproductibilité bien que les zones indentées soient différentes. En augmentant la charge de 50 à $90 \mathrm{~N}$ le comportement devient plus homogène, et on observe pour le Zerodur ${ }^{\circledR}$ des discontinuités sur la courbe forcedéplacement pendant la mise en charge. Ces phénomènes appelés pop-in correspondent à une propagation instable de la fissure. L'allure des courbes force déplacement met en évidence le comportement élasto-plastiques des matériaux [31]. Nous remarquons que la déformation plastique du verre flotté est supérieure aux autres verres. Pour une charge de $90 \mathrm{~N}$ par exemple la pénétration résiduelle du verre flotté est de l'ordre $19 \mu \mathrm{m}$ alors qu'elle n'est que de 16,5 et $125 \mu \mathrm{m}$ pour les verres (flint et crown) et le Zerodur ${ }^{\circledR}$, respectivement.

Le premier pop-in apparait pour le verre Zerodur ${ }^{\circledR}$ pour une charge de $50 \mathrm{~N}$ et plusieurs pop-in successifs sont observés sur la courbe force-pénétration pour des charges supérieure à 200 N. Aucun pop-in n'est repéré sur la courbe charge-décharge pour le verre crown alors qu'ils apparaissent sur le verre flotté et le verre flint vers $300 \mathrm{~N}$.

La Figure 11 représente la variation de la ténacité $K_{I C}$ en fonction de la charge $P$ respectivement pour le verre crown K5, flint lourd KzSFN5, flotté et Zerodur ${ }^{\circledR}$. La ténacité pour ce dernier est légèrement supérieure à celle des autres verres, ce qui est constaté clairement sur les micrographies illustrées dans la Figure 8.

Globalement, pour les échantillons utilisés, et à travers l'écart type nous remarquons que le taux de dispersion des résultats calculée par les quatre relations ne dépasse guère $4 \%$.

La Figure 12 montre l'évolution de la charge $P$ en fonction de la longueur de la fissure pour les quatre verres. La charge appliquée est proportionnelle à $c^{-3 / 2}$, indépendamment de l'évolution de forme des fissures, ce qui correspond à une ténacité constante, signe que le polissage n'a pas introduit de contraintes résiduelles notables. 


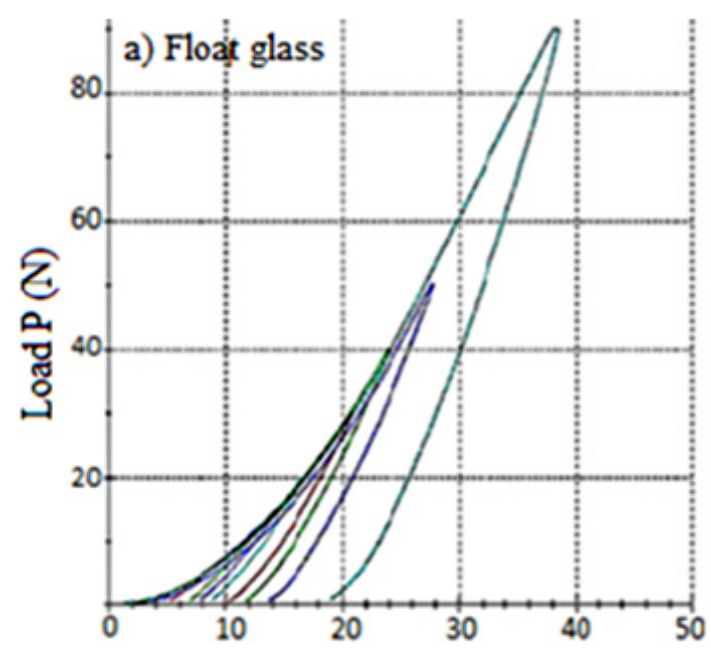

Penetration depth $(\mu \mathrm{m})$

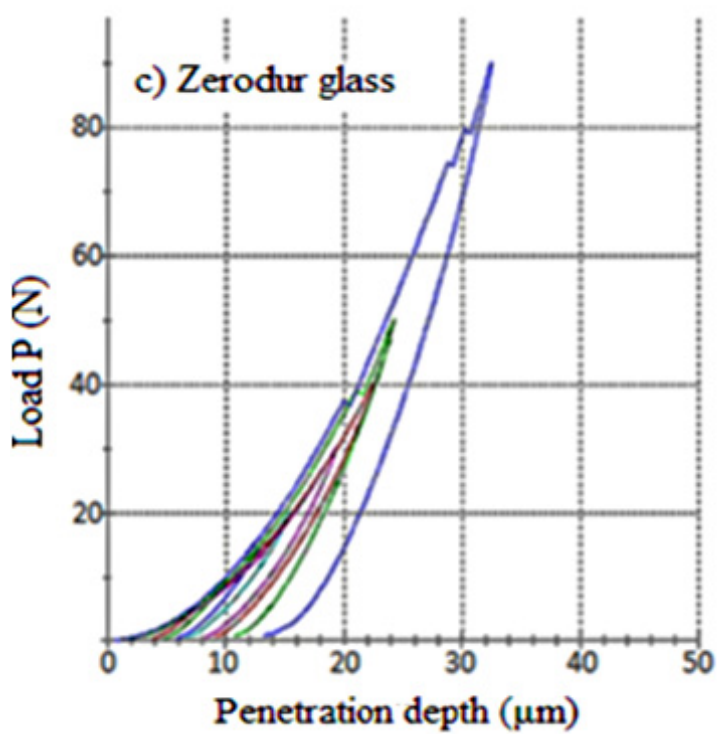

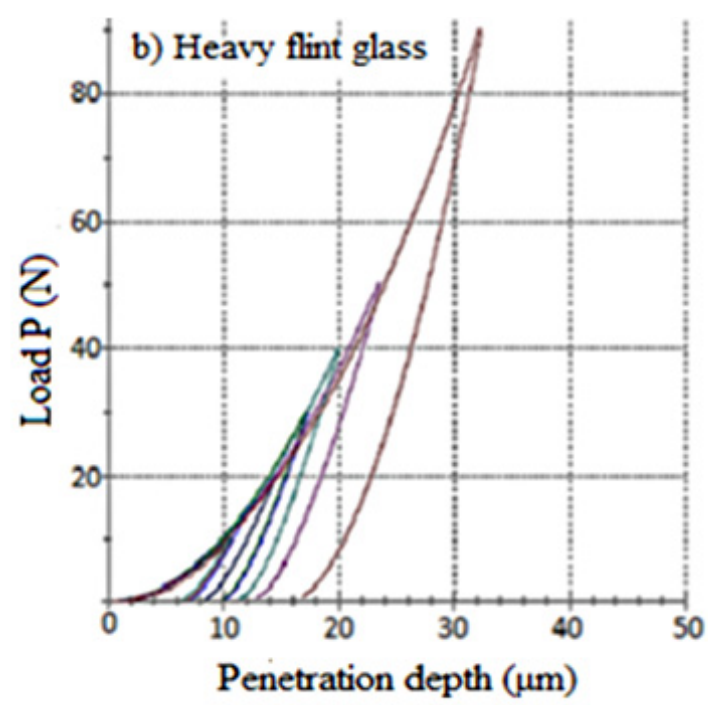

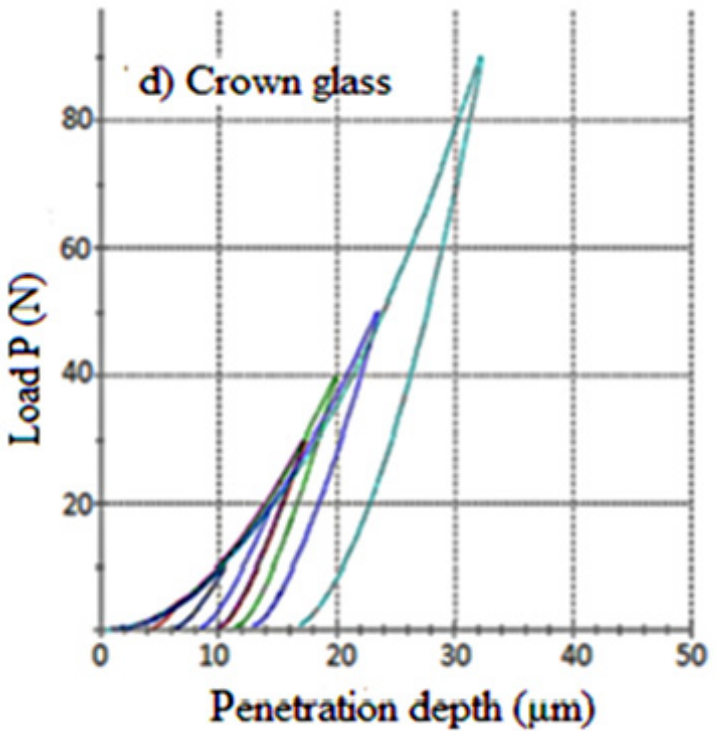

Fig. 7. Courbes charge-profondeur d'indentation pour les quatre échantillons de 5 à $90 \mathrm{~N}$.

Fig. 7. Glasses load-displacement curves from 5 to $90 \mathrm{~N}$.

Tableau 3. Valeurs moyennes de la ténacité, du Module d' Young et de la dureté Vickers des quatre échantillons utilisés. Table 3. Fracture toughness, Young's modulus and Vickers hardness average values of the glasses.

\begin{tabular}{ccccccc}
\hline \multirow{2}{*}{ Glasses } & Young's modulus & Vickers hardness & \multicolumn{3}{c}{ Fracture toughness $K_{I C}\left(\mathrm{MPa} \cdot \mathrm{m}^{1 / 2}\right)$} \\
\cline { 2 - 7 } & $E(\mathrm{GPa})$ & $H(\mathrm{GPa})$ & Anstis & Laugier & Tanaka & Niihara \\
\hline Flotté & $68,80 \pm 1.04$ & $6,2 \pm 0,2$ & $1,02 \pm 0,02$ & $0,73 \pm 0,04$ & $1.09 \pm 0,04$ & $1,05 \pm 0,03$ \\
Crown K5 & $57,43 \pm 1.16$ & $6,15 \pm 0,3$ & $0,83 \pm 0,04$ & $0,54 \pm 0,02$ & $0,85 \pm 0,03$ & $0,87 \pm 0,04$ \\
Flint KZSFN5 & $73,64 \pm 0.91$ & $5,63 \pm 0,18$ & $0,47 \pm 0,02$ & $0,24 \pm 0,02$ & $0,51 \pm 0,02$ & $0,72 \pm 0,02$ \\
Zerodur & $88,07 \pm 0,98$ & $6,79 \pm 0,21$ & $0,93 \pm 0,01$ & $0,32 \pm 0,04$ & $0,98 \pm 0,02$ & $1.08 \pm 0,04$ \\
\hline
\end{tabular}




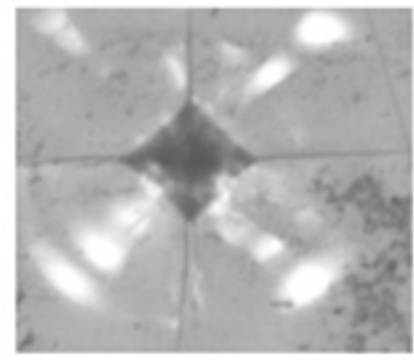

$5 \mathrm{~N}$

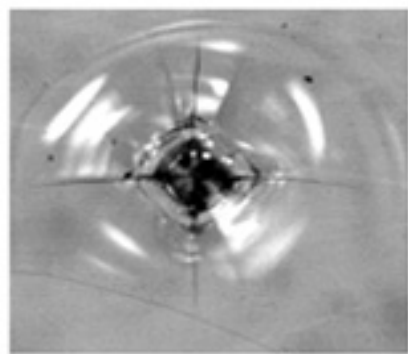

$20 \mathrm{~N}$

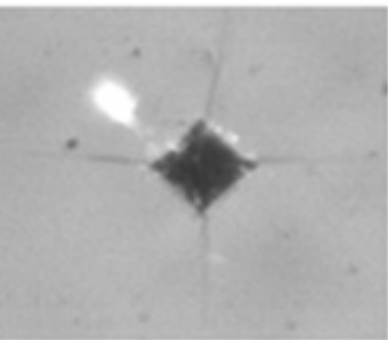

$5 \mathbf{N}$

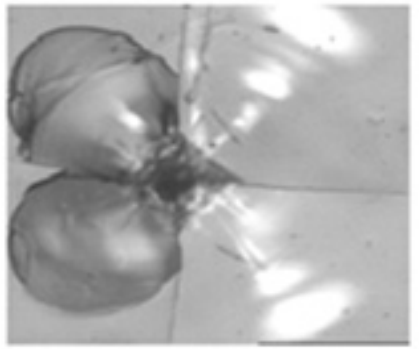

$20 \mathrm{~N}$

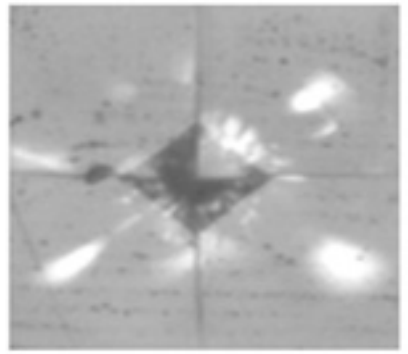

$10 \mathrm{~N}$

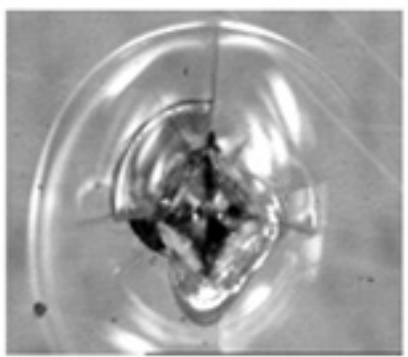

$30 \mathrm{~N}$

Crown K5 glass : Scale $100 \mu \mathrm{m}$

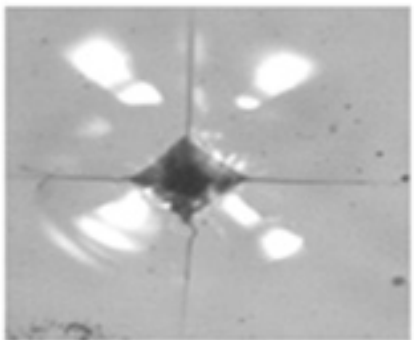

$10 \mathrm{~N}$

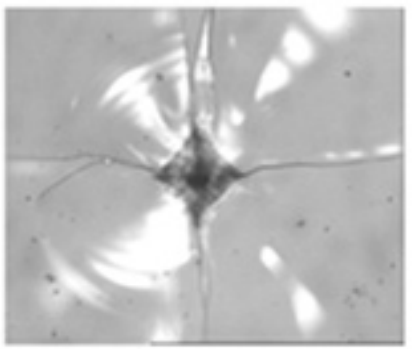

$30 \mathrm{~N}$

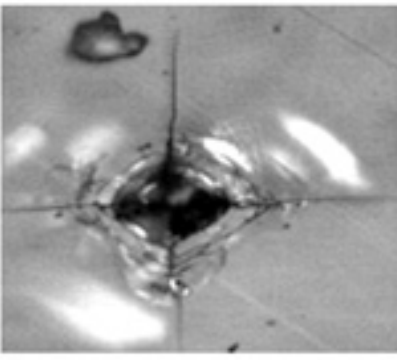

$15 \mathrm{~N}$

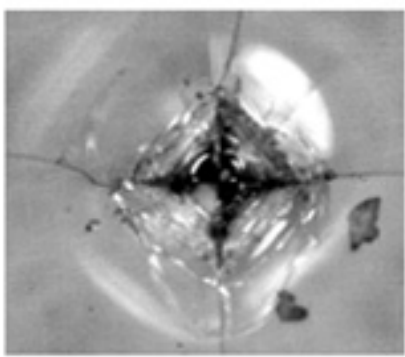

$40 \mathrm{~N}$

\section{.}

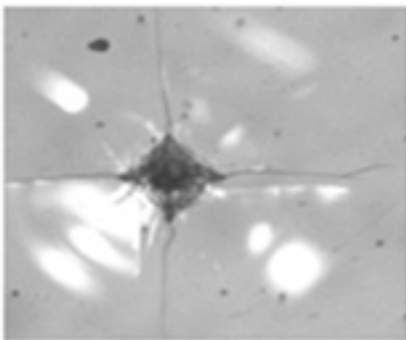

$15 \mathrm{~N}$

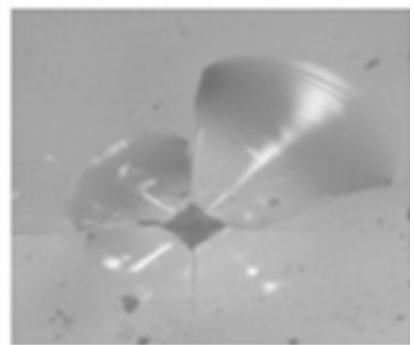

$40 \mathrm{~N}$

Heavy flint glass : Scale $120^{\circ} \mu \mathrm{m}$

Fig. 8. Empreintes d'indentations sur les quatre verres étudiés.

Fig. 8. Glasses load-displacement curves from 5 to $90 \mathrm{~N}$. 


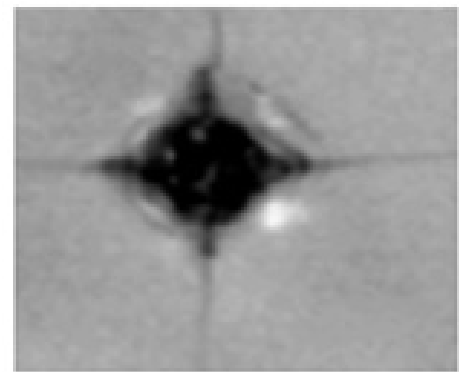

$5 \mathbf{N}$

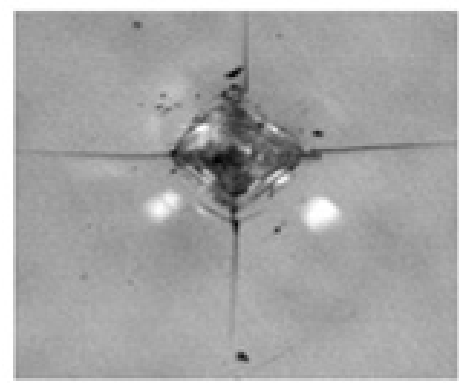

$20 \mathrm{~N}$

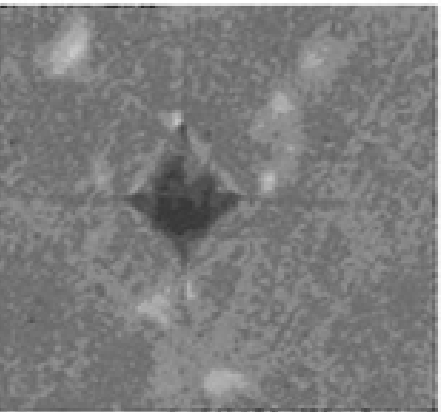

$5 \mathrm{~N}$

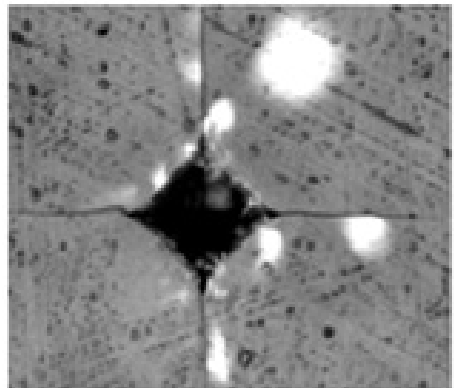

$20 \mathrm{~N}$

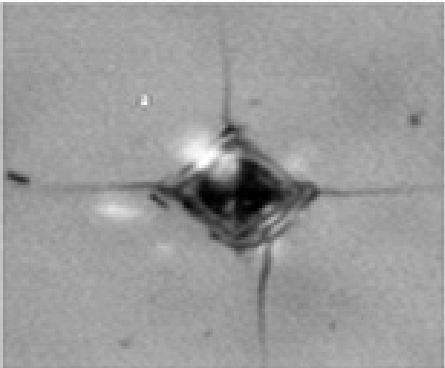

$10 \mathrm{~N}$

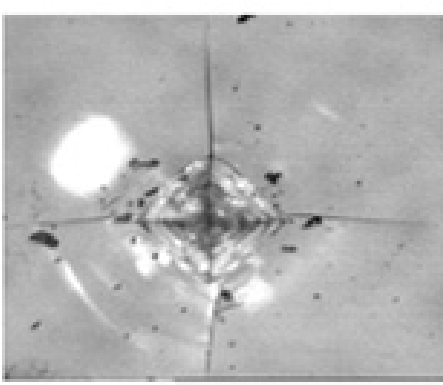

$30 \mathrm{~N}$

Zerodur glass : Scale $120 \mu \mathrm{m}$

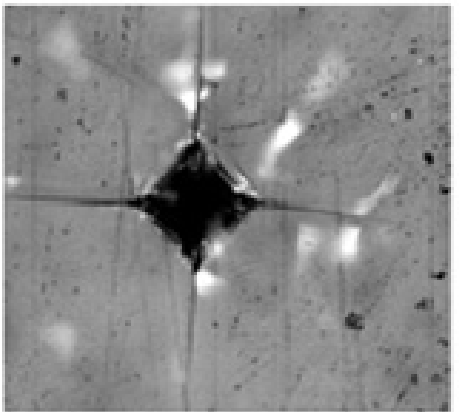

$10 \mathrm{~N}$

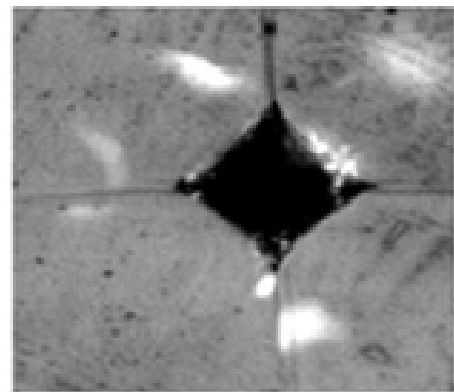

$30 \mathrm{~N}$

\section{a}

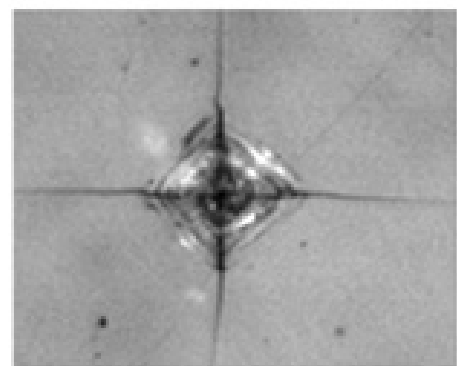

$15 \mathrm{~N}$

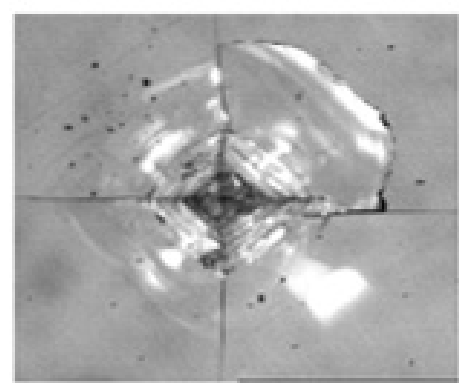

$40 \mathrm{~N}$

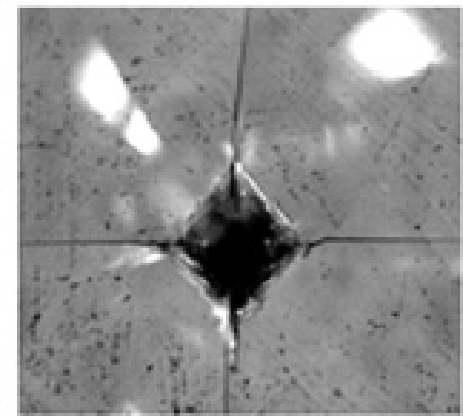

$15 \mathrm{~N}$

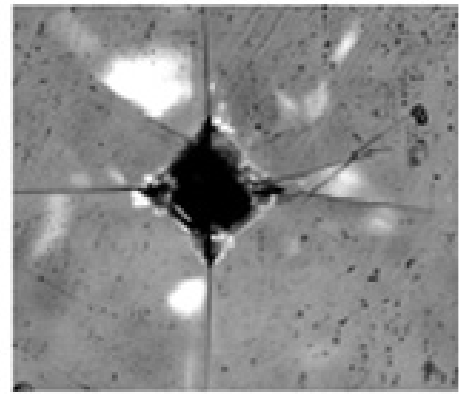

$40 \mathrm{~N}$

Float glass : Scale $100 \mu \mathrm{m}$

Fig. 8. Suite.

Fig. 8. contineud. 

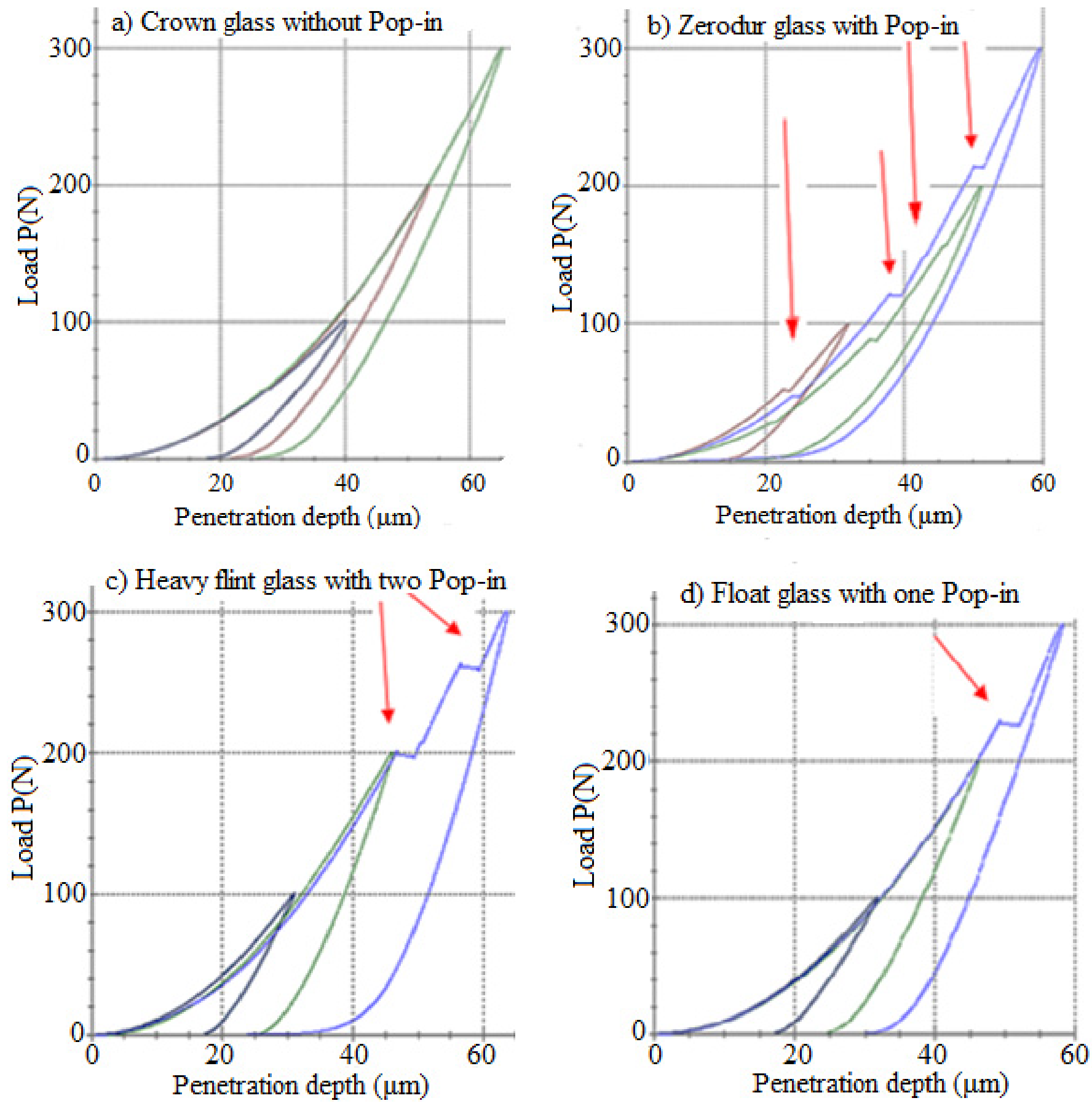

Fig. 9. Courbes charge-profondeur d'indentation pour les quatre échantillons et des charges maximales entre [100-300] N. Fig. 9. Load-displacement curves for the four glasses for a force range between [100-300] N. 


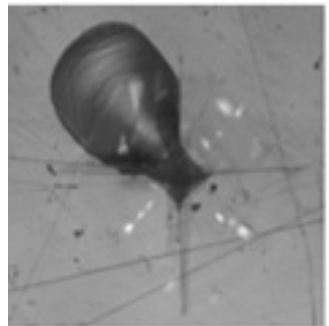

$100 \mathrm{~N}$

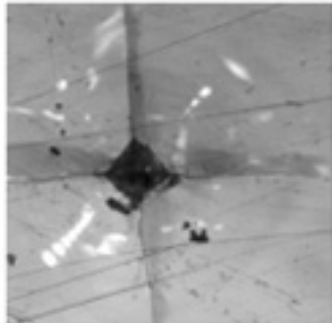

$200 \mathrm{~N}$

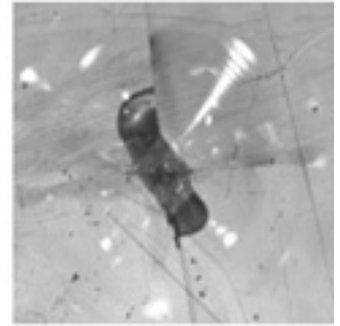

$300 \mathrm{~N}$

Float glass : Scale $400 \mu \mathrm{m}$

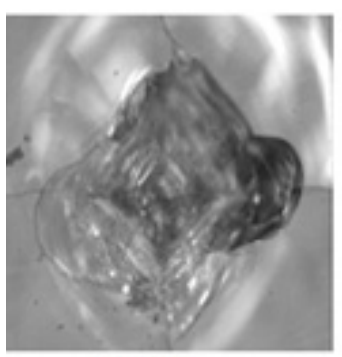

$100 \mathrm{~N}$

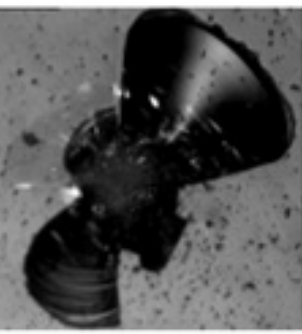

$100 \mathrm{~N}$

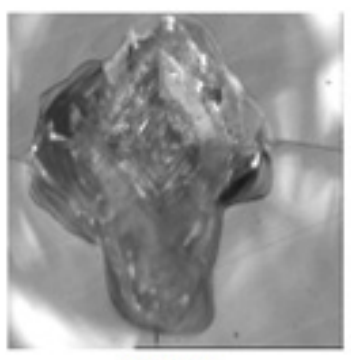

$200 \mathrm{~N}$

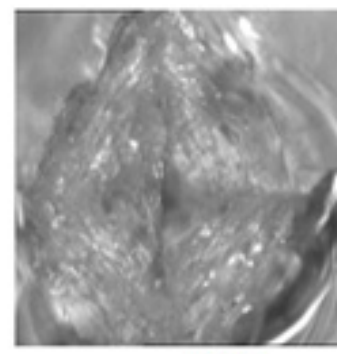

$300 \mathrm{~N}$

Crown $\mathrm{K} 5$ glass : Scale $500 \mu \mathrm{m}$

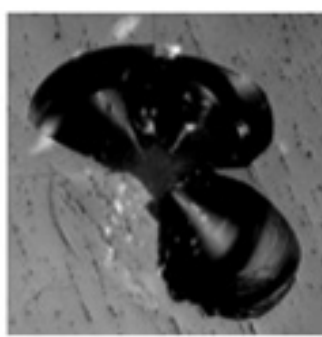

$200 \mathrm{~N}$

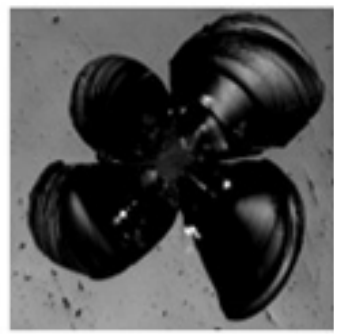

$300 \mathrm{~N}$

Heavy flint glass : Scale $500 \mu \mathrm{m}$

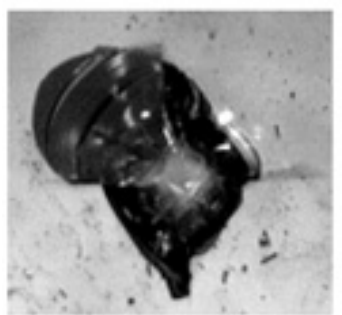

$100 \mathrm{~N}$

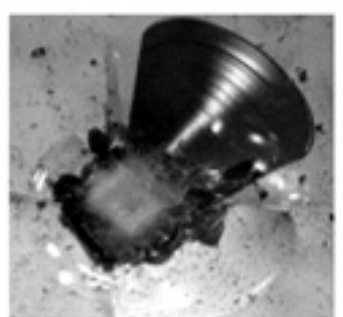

$200 \mathrm{~N}$

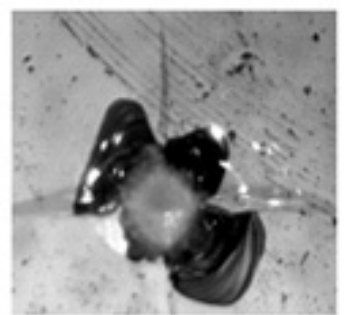

$300 \mathrm{~N}$

Zerodur glass : Scale $500 \mu \mathrm{m}$

Fig. 10. Empreintes d'indentation sur les quatre verres étudiés.

Fig. 10. Vickers indentation imprint on the four studied glasses. 

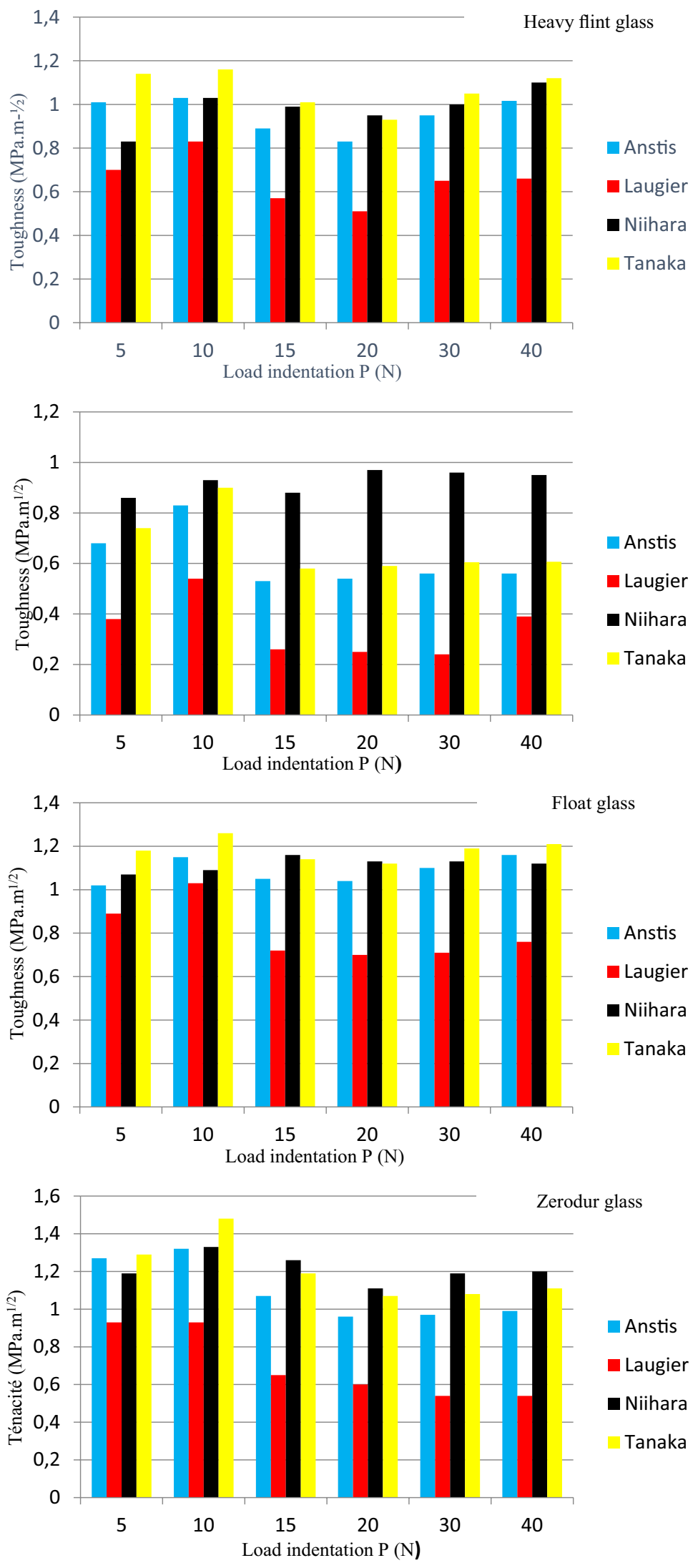

Fig. 11. Variation de la ténacité $\left(K_{I c}\right.$ des quatre types de verre en fonction de la charge appliquée $P$. Fig. 11. Glasses fracture toughness $\left(K_{I c}\right)$ variation according to the applied load $P$. 

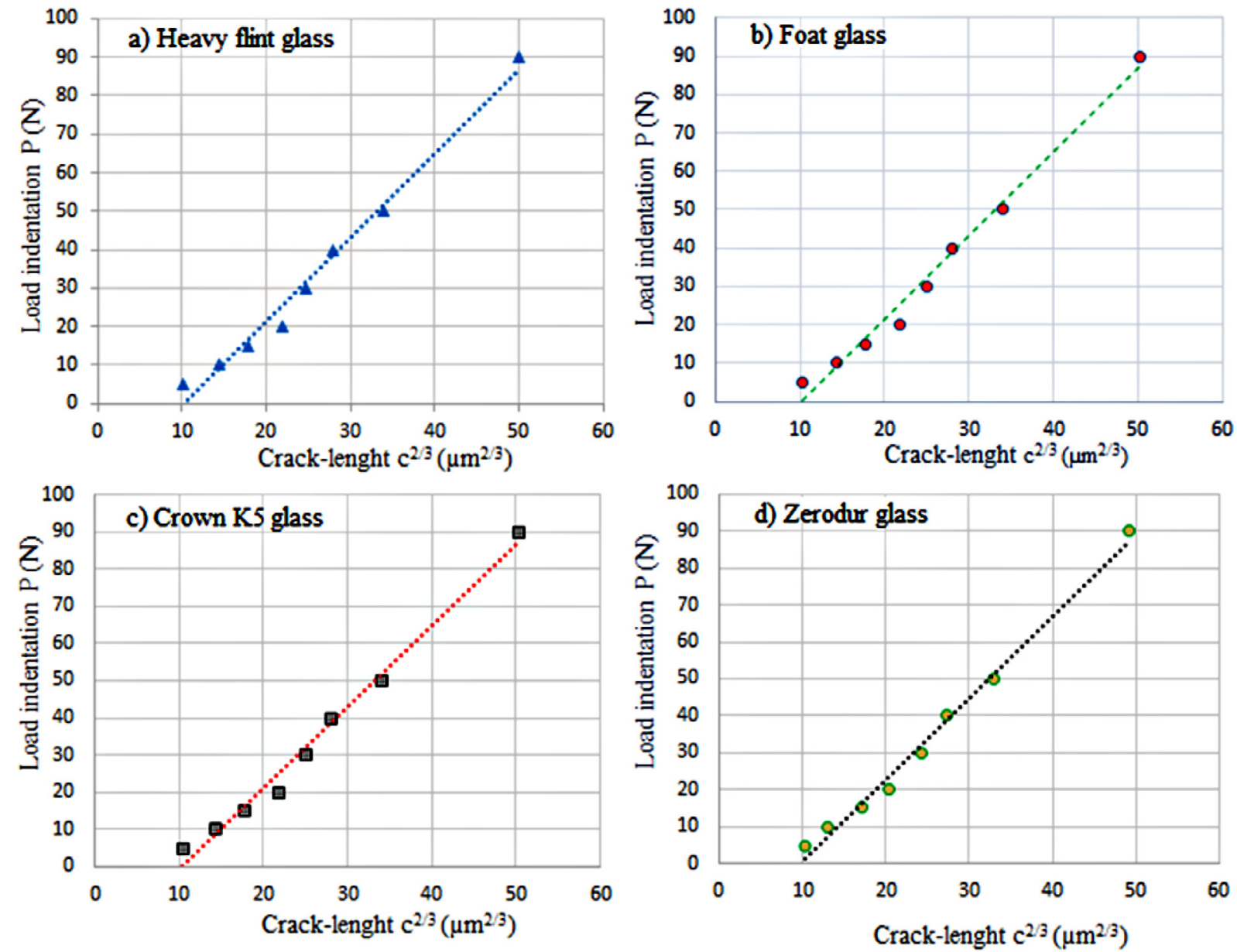

Fig. 12. Évolution de la charge $P$ en fonction de longueur des fissures pour les quatre échantillons étudiés.

Fig. 12. Evolution of the load $P$ as a function of the crack length for the four studied glasses.

\section{Conclusion}

Les résultats obtenus dans le processus de finition permettent de préciser le rôle primordial des grains abrasifs liés dans les processus de rodage et de polissage des surfaces des matériaux fragiles, Le polissage par des grains abrasifs liés montre une qualité de surface meilleure par rapport au polissage par grains abrasifs libres et permet d'obtenir des valeurs de rugosité $R a$ et $R q$ de l'ordre de trente à quarante nanomètres. Néanmoins le verre crown permet d'obtenir une rugosité réduite de $30 \mathrm{~nm}$ comparée à celle du flint et Zerodur, qui est l'ordre de $40 \mathrm{~nm}$.

Le comportement en dureté des verres indentés a été comparé à l'aide des courbes force-déplacement obtenues lors de l'essai instrumenté, et une étude comparative de différentes équations a été faite pour le calcul de la ténacité des quatre verres utilisés.

En effet, d'après les courbes charge-déplacement, nous remarquons que la pénétration résiduelle après déchargement dans le cas du verre flotté est supérieure aux autres verres, elle correspond $19 \mu \mathrm{m}$ et $32 \mu \mathrm{m}$ respectivement pour les charges sélectionnées $90 \mathrm{~N}$ et $300 \mathrm{~N}$, et elle n'est que de $(16,5 \mu \mathrm{m}$ et $26 \mu \mathrm{m}),(16,5 \mu \mathrm{m}$ et $28 \mu \mathrm{m})$ et $(12,5 \mu \mathrm{m}$ et $24 \mu \mathrm{m})$ pour les verres crown, flint et le Zerodur ${ }^{\circledR}$, respectivement.
En plus, les tailles des empreintes et fissures radiales augmentent avec la charge. En appliquant des charges allant de 0 à $90 \mathrm{~N}$, on remarque une bonne reproductibilité sur les courbes charge-déplacement, bien que les trois courbes correspondent aux verres flottés, crowns et flints, montrent un comportement homogène, par contre sur le verre Zerodur, présente une discontinuité sur la courbe force-déplacement pendant la mise en charge où le phénomène de pop-in apparait, ce qui corresponde à une propagation instable de la fissure. Ce phénomène apparait successif pour ce verre sur la courbe charge-décharge pour des grandes charges, et nous remarquons aucun popin sur la courbe du verre crown qui possède une composition riche en silice comparée aux autres verres, mais les pop-in apparaissent sur le verre flotté et le verre flint vers $300 \mathrm{~N}$.

La ténacité $K_{I C}$ a été calculée par la relation de Laugier et de Niihara, elle présente respectivement des intervalles comprises entre 23 et $61 \%$ et entre 4 et $25 \%$; tandis que les valeurs de la ténacité trouvées par la relation de Tanaka et celle d'Anstis sont inférieures à $10 \%$.

Les valeurs moyennes de la ténacité trouvés par la relation d'Anstis du verre crown K5, flotté et Zerodur sont assez grandes comparées à celle du flint, elles 
correspondent respectivement $0,83,1,02,0,93$ et 0,47 pour le verre flint.

En conséquence, la relation d'Anstis peut être choisie pour le calcul de la ténacité $K_{I C}$ des matériaux fragiles (verres sodocalciques, verres optiques ou verres céramiques), puisque cette dernière présente moins de dispersion par rapport aux autres relations et est celle qui est la plus souvent utilisée dans la littérature, ce qui permet de comparer la ténacité de différents matériaux entre eux.

\section{Références}

[1] A. Iost, Rev. Métal. 110 (2013) 215-233

[2] G.A. Gogots, Ceram. Int. 39 (2012) 3293-3300

[3] H.H. Karow, Fabrication methods for precision optics, Wiley-interscience ISBN0-471-70379-6 USA, 2004

[4] D.S. Glancy, M.J. Damgaard, A study in the dynamics and wear of rigid disc systems, 24th Annuals convention of the international metallographic Society in Monterey, California USA, August 1, 1991, p. 3

[5] K. Phillips, G.M. Crimes, T.R. Wilshaw, Wear 41 (1977) 327-350

[6] S.D. Jacobs, S.R. Arrasmith, I.A. Kozhinova, L.L. Gregg, A.B. Shorey, H.J. Romanofsky, D. Golini, W.I. Kordonski, P. Dumas, S. Hogan, Am. Ceram. Soc. Bull. (1999) 42-48

[7] W.I. Rupp, Appl. Opt. (1972) 2797

[8] G.F. Vander Voort, Metallography Principles and Practice, Mc Graw Hill Inc., p. 100, 1982

[9] A.A. Tesar, B.A. Fuchs, Removal rates of fused silica with cerium oxide and pitch polishing, in Advanced Optical Manufacturing and Testing II, V.J. Doherty, Ed. Proc. Soc. Photo-Optics. Instrument. Eng. 1531 (1991) 80-90

[10] D. Golini, J.C. Lamropoulos, P.D. Funenbush, Appl. Opt. 35 (1996) 4448-4462
[11] H.H. Pollicov, Opt. Photon. News 6 (1994) 15-190

[12] J. Liedes, J. Opt. Soc. 1 (1996) 1917-1983

[13] J.C. Lambropoulos, Su Xu, Tong Fang, Appl. Opt. 36 (1997) 1501-1515

[14] D. Bouzid, U. Jungstand, V. Herold, Glass Technol. 42 (2001) 60-62

[15] J.P. Marioge, Surface optique : Méthodes de fabrication et de contrôle, recherches, EDP Sciences France, 2000, pp. 26-33

[16] R. Komanduri, Ann. CIRP 46 (1997) 545-96

[17] E. Brinksmeie, Precision Eng. 30 (2006) 325-336

[18] J. Luo, Integrated modeling of chemical mechanical planarization/polishing (CMP) for integrated circuit fabrication: from particle scale to die and wafer scales, Doctorate Thesis, University of California, Berkeley, 2003

[19] C.B. Ponton, R.D. Rawlings, Mater. Sci. Tech. 5 (1989) 865-872

[20] C.B. Ponton, R.D. Rawlings, Mater. Sci. Tech. 5 (1989) 961-976

[21] K.K.Bamzai, P.N. Kotru, Mater. Sci. Tech. 16 (2000) 405- 410

[22] D.G. Bhat, J. Am. Ceram. Soc. 64 (1981) C165-C-166

[23] E. Le Bourhis, Glass mechanics and technology, 2nd, Wiley.VCH, 2014

[24] J. Petzoldt, Jenaer Glaswerk Schott Genossen, Mainz, Fed. Rep. Ger. Glastechnische Berichte 43 (1970) 127-34

[25] W. Pannhorst, Am. Ceram. Soc. Bull. 82 (2003) 93019306

[26] G. Mueller, Glass-Ceramic Materials. Schott Information, 1972, pp. 1-7

[27] G.R. Anstis, P. Chantikul, B.R. Lawn, J. Am. Ceram. Soc. 64 (1981) 533-538

[28] K.Tanaka, J. Mater. Sci. Lett. 22 (1987) 1501-1508

[29] K. Niihara, R. Morena, D.P.H. Hasselman, J. Mater. Sci. Lett. 1 (1982) 13-16

[30] M.T. Laugier, J. Mater. Sci. Lett. 6 (1987) 355

[31] A. Chorfa, M. Hamidouche, M.A. Madjoubi, F. Petit, Int. Rev. Mech. Eng. 1 (2007) 105 